\title{
In Silico Analysis Brucella OMPs and CagA for Expansion of a Subunit Vaccine Candidate Versus Brucellosis
}

\author{
Amir Hosseinabadi ${ }^{1,2}$, Mohsen Korani ${ }^{2,3}$, Davoud Esmaeili ${ }^{1,2^{*}}$ \\ 'Department of Microbiology and Applied Microbiology Research Center, Systems Biology and Poisonings Institute, Baqiyatallah \\ University of Medical Sciences, Tehran, Iran \\ ${ }^{2}$ Applied Virology Research Center, Baqiyatallah University of Medical Sciences, Tehran, Iran \\ ${ }^{3}$ Chemical Injuries Research Center, System Biology and Poisoning Institute, Baqiyatallah University of Medical Sciences, Tehran, \\ Iran \\ ${ }^{4}$ Department of Biochemistry, Faculty of Medicine, Baqiyatallah University of Medical Sciences, Tehran, Iran
}

Corresponding Author: Davoud Esmaeili, PhD, Associate Professor, Department of Microbiology and Applied Microbiology Research Center, Systems Biology and Poisonings Institute, Baqiyatallah University of Medical Sciences, Tehran, Iran. Email: esm114@gmail.com

Received September 6, 2018; Accepted January 28, 2019; Online Published March 15, 2019

\begin{abstract}
Brucellosis is one of the current zoonotic diseases, still a health hazard in many countries, and progressive research programs are necessary to control and eradicate this disease in endemic areas. Outer membrane proteins (OMPs) are capable of allocating immunity and protection antigen in mice. CagA is an immunogenic protein of Helicobacter pylori. Because OMPs are part of Brucella spp, they have been conferred as potential immunogenic and protective antigens. In this study, the gene sequence which encoding TN-OMPs was obtained from GenBank. To estimate the 3D structure of the protein, modeling, prediction of secondary and tertiary structure, immunogenicity, allergenic sites and antigenic B-cell and T-cell epitopes were performed. The conserved domain of proteins was obtained and the epitopes of TN-OMPs were capable of inducing both B-cell and T-cell mediated immune responses. CagA is a cellular immunogenic protein that induces a Th1 response. The OMPs of Brucella could be used as a suitable vaccine candidate versus brucellosis.

Keywords: Brucellosis, OMPs, CagA, Insilco Analysis, Vaccine

Citation: Hosseinabadi A, Korani M, Esmaeili D. In silico analysis Brucella OMPs and CagA for expansion of a subunit vaccine candidate versus brucellosis. Int J Med Rev. 2019;6(1):14-20. doi:10.29252/IJMR-060103.
\end{abstract}

\section{Introduction}

Brucella spp. are gram-negative and facultative intracellular bacteria, an a-2 proteobacteria subdivision, which cause brucellosis, abortion in animals, and Malta fever in humans. ${ }^{1}$ Brucellosis is the most common zoonotic disease in the world, found mainly around the Mediterranean Sea, South America, and the Middle East. More than 500000 new cases of brucellosis are reported annually. ${ }^{2}$ The diverse symptoms of this zoonosis disease in humans contracted, through contact with a contaminated animal, is prostatitis, spondylitis, encephalitis, orchitis, and arthritis. ${ }^{3}$ Three families of the outer membrane protein (OMP) of Brucella have been identified: group 1 (94 or $88 \mathrm{kDa})$, group $2(36-38 \mathrm{kDa})$, and group 3 (31-34 and 25-27 kDa). ${ }^{4}$ The OMP of Brucella have been identified as the protective immunogenicity, and currently, research for the development of a subunit vaccine is increasing through the exploitation of the outer membrane and other ribosomal proteins. ${ }^{5}$ The OMP28 (bp26) of Brucella has been identified as an immunodominant periplasmic antigen. ${ }^{6}$ It is highly conserved in the Brucella genus and capable of inducing both humoral and cellular immunity. ${ }^{7}$ The OMP31 expresses all species of Brucella abortus. ${ }^{8}$ The OMP31 of Brucella melitensis had 34\% homology with OMP25 of Brucella abortus, and the C-terminal OMP31 of B. melitensis has phenylalanine that is essential for bacterial surface translocation. ${ }^{9}$ The cytotoxin-associated gene A (CagA) is a $120-128 \mathrm{kDa}$ part of the cag pathogenicity island (cagPAI) that has been identified as an immunodominance protein, the 5 '-region missing phosphorylation motifs (EPIYA-A, $-B,-C$, or $-D)$ of which are the cancer domain. ${ }^{10}$ Currently, brucellosis is controlled in animals by using the live attenuated vaccines of Brucella as melitensis Rev 1 and B. abortus S19 and RB51.11 The disadvantages of these vaccines are that they are unsafe for use in humans, they cause abortions in sheep, the immunization is short-lived, and pathogenicity returns. ${ }^{12}$ Since Brucella is intracellular bacteria and immunity against Brucella are both cellular and humoral immune responses, ${ }^{13}$ most vaccine candidates can induce antibodies, CD4+, CD8+, and especially IFN- $\gamma$, the main role of which is to induce macrophage activity. ${ }^{14}$ A 48-72 amino acid of OMP31 of

Copyright (C) 2019 The Author(s). This is an open-access article distributed under the terms of the Creative Commons Attribution License (http:// creativecommons.org/licenses/by/4.0), which permits unrestricted use, distribution, and reproduction in any medium, provided the original work is properly cited. 
Brucella does not elicit a humoral response, but the cellular and Th-1 are required. ${ }^{15}$ The antibody responses of OMP31 and bp 26 obtain low levels, but IFN- $\gamma$ is induced by the epitope of bp26: 6-8-11-12 and epitope of OMP31: $12 .{ }^{16}$ In the current study, OMP31, bp26 of Brucella, and cellular immunogenic CagA of Helicobacter pylori were analyzed in order to develop a subunit-appropriate vaccine against brucellosis.

\section{Materials and Methods}

Sequence Analysis and Segment Selection

Sequences of OMP31, OMP28, and CagA were obtained from GeneBank using the software http://www.uniprot.org/ uniprot/P0A3U8. To identify a fragment common among all sequences, multiple sequence alignments to identify a conserved region in each sequence were performed using Clustal W software (http://www.ebi.ac.uk/Tools/clustalw2). The total amino acids of OMPS were selected. Furthermore, the presence of appropriate epitopes was confirmed with other web-based B-cell and T-cell epitope prediction algorithms in single or assembled forms of selected segments. The VaxiJen server was used to predict the immunogenicity of the whole antigen and its subunit vaccine.

\section{Physicochemical Parameters}

Extinction coefficient, theoretical isoelectric point (pI), molecular weight, the total number of positive and negative residues, half-life, instability index, aliphatic index and grand average hydropathy (GRAVY), and the physicochemical parameters were computed using Expasy's ProtParam (http:// us.expasy.org/tools/protparam.html). ${ }^{17}$

\section{Protein Secondary Structure}

The secondary structure of the protein was predicted using the GOR secondary structure prediction method. ${ }^{18}$ To conduct sequence analysis and predict protein structure and function, such as low-complexity regions, regions lacking regular structure, secondary structure, solvent accessibility, transmembrane helices, coiled-coil regions, disulfidebonds, sub-cellular localization, and functional annotations, PredictProtein server ${ }^{19}$ was used.

\section{Prediction of Antigenic B-cell Epitopes}

Three web-based B-cell epitope prediction algorithms (Bcepred http://www.imtech.res.in/-raghava/bcepred/, Continuous B-cell epitopes prediction methods based on physicochemical properties on a non-redundant dataset, and Discotope Server http://www.cbs.dtu.dk/services/DiscoTope/) were used to predict discontinuous B-cell epitopes from three-dimensional protein structures in order to analyze amino acid sequences. Briefly, chimeric proteins were first analyzed for continuous B-cell epitopes using Bcepred, and all predicted B-cell epitopes (20 mers) having a BcePreds cutoff score $>0.8$ were selected. Then, the Discotope server was used to predict discontinuous B-cell epitopes. Conformational B-cell epitope from the primary sequence was predicted with web server CBTOPE. ${ }^{20}$ Surface-exposed B-cell epitope sequences having a cutoff value for BcePreds
$(>0.8)$ were selected and analyzed further using VaxiJen (threshold=0.4, ACC output) to check the antigenicity. In addition, the location of conformational epitopes on protein surfaces was defined by Research software. ${ }^{21}$

\section{Prediction of T-Cell Epitopes}

To identify common epitopes that bind to both MHC class molecules and to count the total number of interacting MHC alleles, Propred-1 (MHC Class-I alleles) $)^{19-22}$ and Propred (MHC Class-II alleles) $)^{21,22}$ servers utilizing amino acid position coefficients were used. The half maximal (50\%) inhibitory concentration (IC50) and antigenicity of common epitopes being predicted by Propred-1 and Propred were calculated using MHCPred server $(20,21,22)$ and VaxiJen, respectively.

\section{Tertiary Structure Prediction}

Further analysis of the synthetic protein 3D structural stability was done by Swiss-PdbViewer for energy minimization. ${ }^{20}$ The Swiss model is an automated modeling software that develops a $3 \mathrm{D}$ structure model of an unknown structure protein based on the sequence homology with the known structured protein. The modeled 3D structures were visualized using Rasmol tool and Accelrys Discovery Studio 2.5.

\section{Protein Solubility Prediction}

The solvent accessibility of different residues was evaluated by DSSP and VADAR (http://vadar.wishartlab.com/).

\section{Allergenic Sites Prediction}

AlgPred was used to analyze the presence of possible allergenic sites. AlgPred allows allergen predication based on the similarity of known epitopes with any region of the protein. Further analysis of allergenicity was done by homology search in SBeing reported as strong immunogens, $(6,7,9)$. The TF protein and two peptides of B. melitensis, 27 and18 amino acids from OMP31 and bp26, respectively, were selected for the present study.

Sequence comparison by ClustalW showed that these sequences were highly conserved among different strains of $\mathrm{B}$. melitensis. The schematic diagram of protein domain structures with linker sites is shown in Figure 1. Linkers, consisting of EAAAK repeats and expected to form a monomeric hydrophobic a-helix, were designed and used to separate the different domains. It has been reported that Glu-Lys+ salt bridges between repeated Ala can stabilize helix formation. Four repeated EAAAK sequences were introduced between different domains for more flexibility and efficient separating. The arrangements of fragment junctions and linked sites are shown in Figure 1. The amino acid was back-translated based on the Escherichia coli host, and the synthetic chimera was analyzed for their codon bias and GC content. The codon adaptation index on the optimized chimeric gene was 0.98 . The percentage of codon having a frequency distribution of 91-100 in the native chimeric gene was $45 \%$, which was significantly improved to $70 \%$ in the optimized gene sequence. The overall GC content, which is 
a measurement of transcriptional and translational efficiency, was improved to 52. Polyadenylation signal, instability elements, and all cis-acting sites that may have a negative influence on the expression rate were removed. Furthermore, the necessary restriction enzyme sites (EcoRI and HindIII) were introduced at the sequence ends for cloning purposes (DAP database). ${ }^{21}$

\section{Results}

Sequence Selection and Construct Design

The results of the Blast analysis showed the sequences of OMP31 (Figure 1) and OMP28 (bp26) (Figure 2). The whole amino acid of OMPs and 841 base form N-terminal of CagA is shown in Figure 3. The results of the sequence analysis showed that OMP25, OMP31, OMP28 (bp26), and CagA should be selected as parts of the chimeric construct.

Primary Sequence Analysis

The physico-chemical characteristics of the OMPs, such as molecular weight, theoretical isoelectric point (pI), instability,

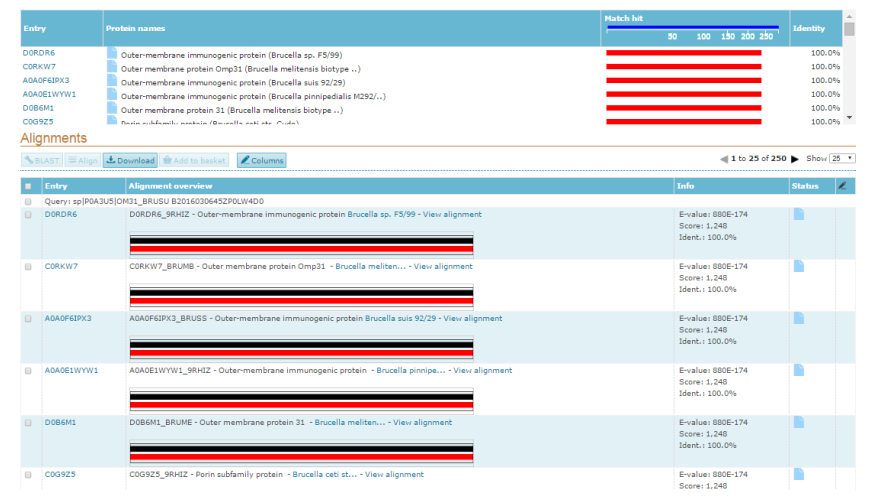

Figure 1. Blast of OMP31.

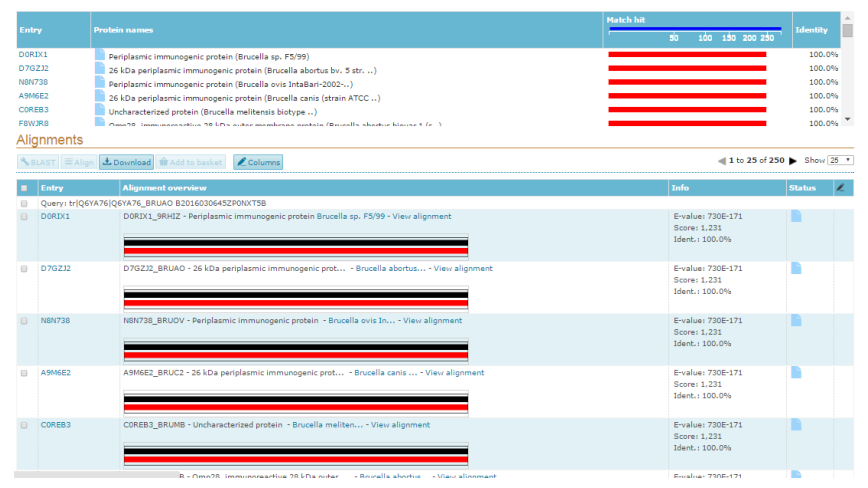

Figure 2. Results in Blast of OMP28 (bp26) and aliphatic which were computed by "ProtParam" and are listed in Table 1. The results of the amino acid composition of the designed protein showed that alanine (20\%), isoleucine $(10.8 \%)$, and proline $(9.2 \%)$ are more abundant than other amino acids. According to the aliphatic index, the designed protein had high thermostability (Table 1 ). The estimated halflife of this sequence was 20 hours (mammalian reticulocytes, in vitro), $30 \mathrm{~min}$ (yeast, in vivo), and more than 10 hours (E. coli, in vivo). The prokaryotic sub-cellular localization of the protein was predicted to be cytosolic. According to the results achieved with Vaxijen and ANTIGENpro software, the chimeric protein might be an antigen. Based on the prediction of APPEL, the construct was not considered as an allergenic domain. Moreover, no experimentally proven IgE epitope was found in this antigen, and no predicted signal peptide was identified in the initial region of the protein sequence. The results of solvent accessibility distribution patterns showed that the mean residue accessible surface area gave a high solvent accessibility value, and the predicted epitopes were located in the accessible region (data not shown).

Prediction of Secondary and Tertiary Structure

The comparison of two prediction methods for assessing the structures of the chimeric protein is shown in Figure 3. This evaluation indicated that the percentage of alpha-helices was greater in OMP31 $(46.38 \%)$ than those of the random coils $(44.93 \%)$ and the extended strand $(8.7 \%)$. The results of the OMP25 showed that helix structures are located in the regions of amino acids 9 to 32, 58 to 87, and 112 to 122 (Figure 3 and Figure 4).

Evaluation of Structural Stability and Validation

The profile of energy minimization which was computed by spdbv (Swiss-PdbViewer) (-6250.604Kcal/mol) showed

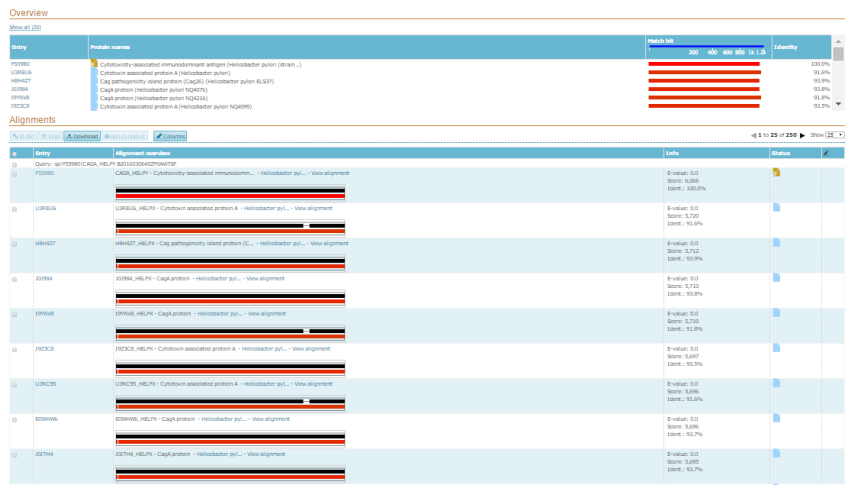

Figure 3. Blast of 841 base primary of CagA.

Table 1. Physico-chemical Properties of OMPs

\begin{tabular}{lcccccc}
\hline & MW (kDa) & Isoelectric Point & Aliphatic index & A280 Extinction Coefficient & Instability index & GRAVY \\
\hline OMP28 & 28 & 8.3 & 70 & 0.9 & 45.51 & 0.193 \\
OMP31 & 31 & 6.7 & 54 & 0.5 & 45.50 & 0.176 \\
OMP25 & 25 & 4.7 & 60 & 0.3 & 33 & 0143 \\
\hline
\end{tabular}




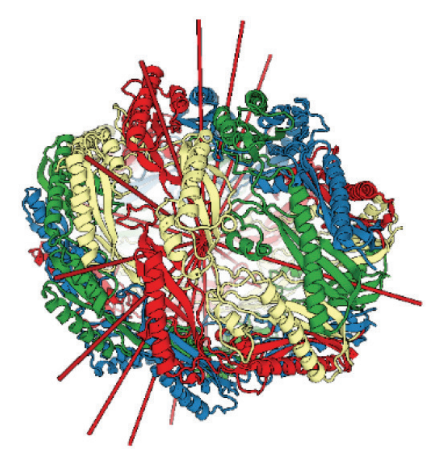

Figure 4. $\mathrm{Ab}$ initio and comparative modeling were employed to predict the 3D structure of OMP28. The result was viewed by Accelrys Discovery Studio Visualizer 2.0 software. The linkers have been displayed in yellow, small loops in green, and large loops in red.

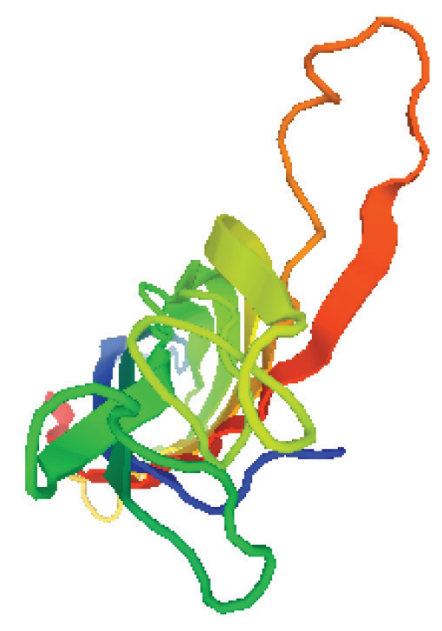

Figure 5. Ab initio and comparative modeling was employed to predict the 3D structure of OMP31.

that protein OMP31 had acceptable stability compared with that of the original structure of each domain. The results of RAMPAGE showed that $81.9 \%$ and $11.8 \%$ of residues are located in the favored and allowed regions, respectively, and only $6.3 \%$ are in the outlier region. The data created by a Ramachandran plot verified the structural stability of the protein (Figure 5).

\section{B-Cell and T Cell Epitope Prediction}

The results of B-cell epitope prediction based on important properties of epitopes, including hydrophilicity, accessibility, flexibility, antigenicity, polarity, and exposed surfaces of both full length and chimeric CagA protein linear B-cell epitopes. The results of the current study also demonstrated that amino acid residues, which were designed as linkers between different domains (amino acid residues 10-16, 60-71, and 115-121), showed no epitope properties.

Proteasomecleavage prediction server (PCPS) demonstrated 52 proteasomal cleavage sites in the protein with a threshold of 0.5. Finally, the prediction of T-cell epitopes using the NetCTL server showed that two T-cell epitopes out of 130 residues are distributed in different sites of the synthetic construct (Figure 6). ${ }^{5}$

\section{Discussion}

In this study, a novel construct of OMPs which is repeated twice (GenBank KM076765) is presented and analyzed (Figures 7-10). The molecular weight of proteins was found as computed by Prot-Param (Figure 10). These linkers also prevent non-native interactions between domains that may interfere with the correct folding and provide structural flexibility (Figure 11), improving protein stability and increasing biological activity. ${ }^{19,20}$ As is known, the pliability of an antigen is an influential parameter in the immunogenicity of any protein exposed to the respective receptors and its recognition by immune cells. Therefore, by enhancing the

\section{Motifs in bms:BR1475 \\ Organism : T00096 \\ Gene: BR1475 \\ Definition : immunoreactive 28 kDa outer membrane protein \\ bma:BR1475 1 MUTRASWFLAASFSTIMLVGAFSL PAFAQENQMTTQPARIAVTGEGMMTASPDMAILMIS 60 \\ pi: DUF 436

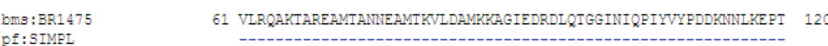 \\ PF:STMPI \\ bms:BR1475 \\ 121 ITGYSVSTSLTVRVRELAIVGKILDESVILGWNQGGDLNIVWDNPGAVINEARKRAVANA. 180 \\ DE: STMPL \\ bmis:BR1475
pf: $:$ IMPL \\ boms: BR1475

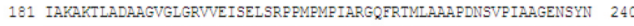 \\ 241 VSVIVVEETK 250}

Figure 6. Amino acid motif of bp26

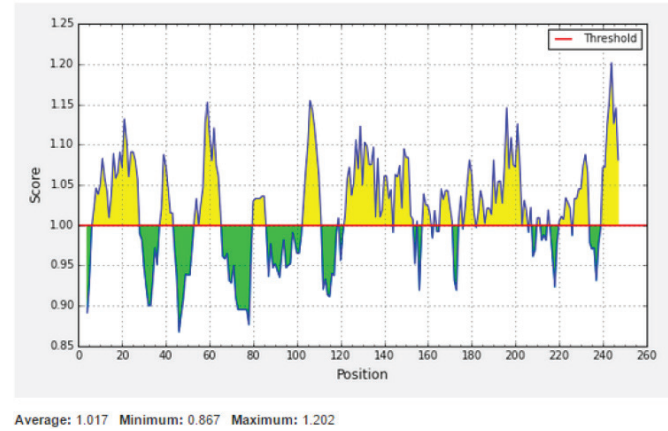

Figure 7. Analysis of Amino Acid Motifs for Induction of Immune Response.

\begin{tabular}{|c|c|c|c|c|}
\hline No. $\$$ & Start क & End $\%$ & Peptide & Length $\%$ \\
\hline 1 & 1 & 3 & MNT & 3 \\
\hline 2 & 27 & 42 & FAQENQ⿻川TTQPARIAV & 16 \\
\hline 3 & 48 & 51 & GMMTAS & 6 \\
\hline 4 & 66 & 78 & KTAREAMTANNEA & 13 \\
\hline 5 & 89 & 99 & AGIEDRDLQTG & 11 \\
\hline 6 & 101 & 101 & I & 1 \\
\hline 7 & 108 & 125 & VYPDDKNNLKEPTITGYS & 18 \\
\hline 8 & 151 & 157 & GVNQGGD & 7 \\
\hline 9 & 159 & 170 & NLVNDNPSAVIN & 12 \\
\hline 10 & 174 & 175 & KR & 2 \\
\hline 11 & 188 & 190 & TLADA & 5 \\
\hline 12 & 192 & 193 & GV & 2 \\
\hline 13 & 204 & 213 & LSRPPMPMPI & 10 \\
\hline 14 & 224 & 240 & AAPONSVPIAAGENSYN & 17 \\
\hline
\end{tabular}

Figure 8. Antigenicity Prediction of bp26 and Antigenic Peptides. 


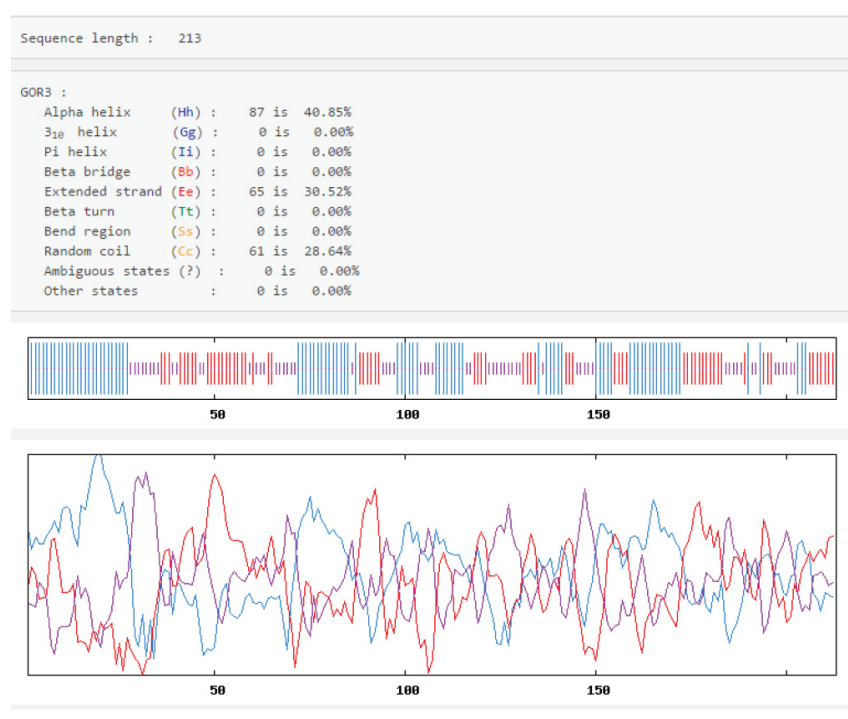

Figure 9. Graphical Results for Secondary Structure Prediction of OMP25; Extended Strand, Purple; Coil, Red; Helix, Blue.

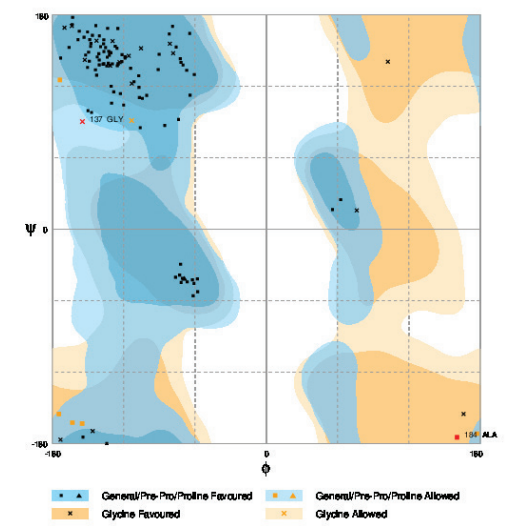

Figure 10. Validation of Protein Structure Using Ramachandran Plot. The Ramachandran plot revealed that $81.9 \%$ of amino acid residues from the modeled structure lie in the most favored region of the plot, and $11.8 \%$ of the residues were in the allowed regions of the plot.

GRAPHICAL RESULT :: SEO 121 to 180

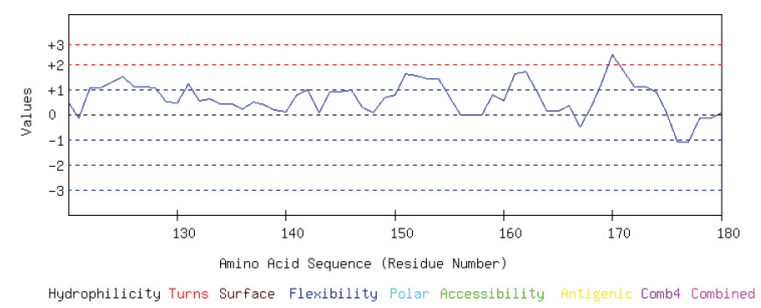

Figure 11. Hydrophilicity and Surface Flexibility of CagA.

structural flexibility and accessibility of this protein by the linker attachment, the second step in antigen improvement is provided (Figures 12 and 13).

The results regarding structural stability and validation showed that most residues of this antigens are in the favored and allowed region, which strongly recommend the stability and validity of the antigen according to the RAMPAGE plot. Therefore, after expression in the bacterial host, its protein could have enough stability and resistance to degradation, which are important points in the immunogenicity of the antigen.

After being assured of having the suitable antigen in terms of B-cell epitope prediction, the next step was to analyze its binding to MHC. For this purpose, the CagA protein was analyzed for MHC class I binding regions. From fifteen consensus regions, nine peptides showed high and intermediate binding affinity to the TAP protein. These selected forms of the antigen, which contain peptides necessary for TAP recognition, can easily be exposed to MHC-I. Therefore, the new OMP antigens formed by adding only those peptides in the selected epitopes, accelerate the research process and induce a high immune response, thus saving time and resources in cases of heterologous gene expression in the prokaryotic host (Figures 12 and 13).

AlgPred software was used to analyze the presence of possible allergenic sites. This program allows allergen predication based on the similarity of known epitopes with any region of the protein (Figures 14-16).

\section{Conclusions}

The current results indicate that epitopes of the OMPs and CagA can induce both B-cell and T-cell mediated immune responses. The protein has proper stability and antigenicity, and it has no allergenicity. In general, data from this study indicates that this protein could be an appropriate immunogen candidate against Brucellosis.

\section{Conflict of Interest Disclosures}

The author declares he has no conflicts of interest.

\section{References}

1. Siadat SD, Salmani AS, Aghasadeghi MR. Brucellosis vaccines: an overview. IntechOpen; 2012. doi:10.5772/38812.

2. Bahador A, Esmaeili D, Mansoori N, Mahdavi M. Protection
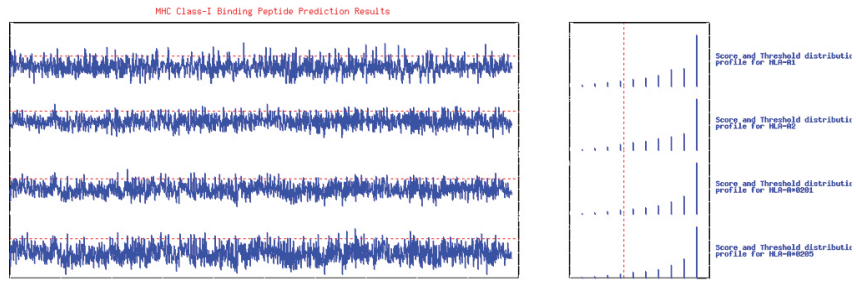

Figure 12. MHC Class I binding Peptide of CagA.
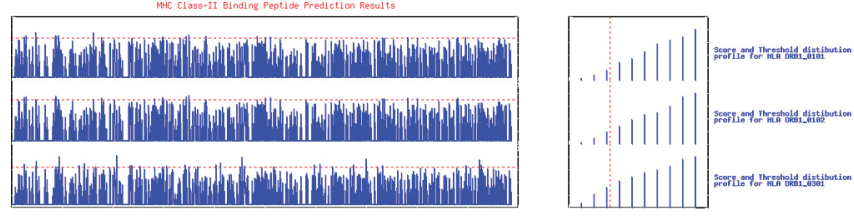

Figure 13. MHC Class II Binding Peptide of CagA 

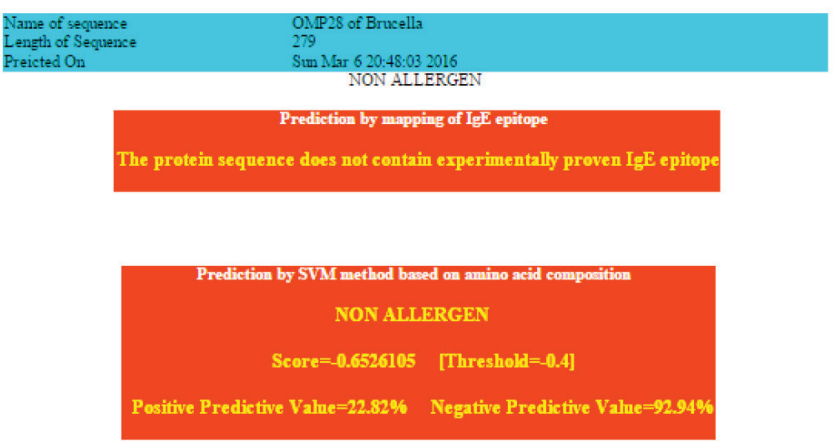

Figure 14. Allergenicity of bp26.

\section{AlgPted: Prediction of Allergenic Proteins and Mapping of IgE Epitopes}
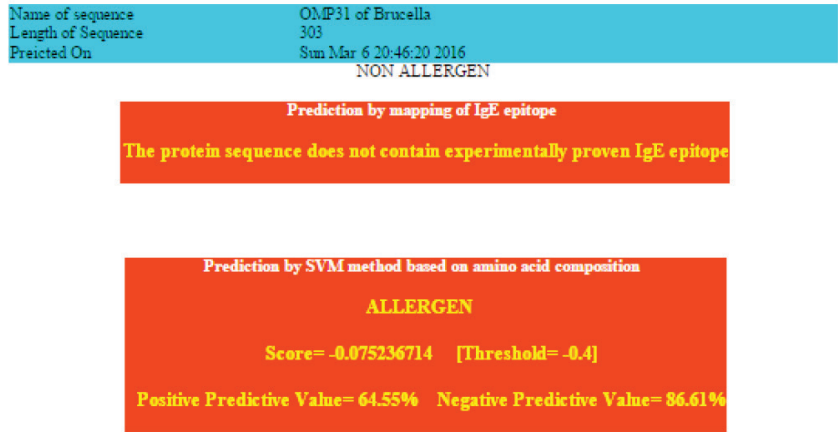

Figure 15. Allergenicity of OMP31.

\begin{tabular}{|c|c|c|c|c|}
\hline No. $\$$ & Start * & End क & Peptide & Length 4 \\
\hline 1 & 1 & 3 & MNT & 3 \\
\hline 2 & 27 & 42 & FAQENQINTTQPARIAV & 16 \\
\hline 3 & 48 & 51 & GMMTAS & 8 \\
\hline 4 & 68 & 78 & KTAREAMTANNEA & 13 \\
\hline 5 & 89 & 99 & AGIEDRDLQTG & 11 \\
\hline 6 & 101 & 101 & I & 1 \\
\hline 7 & 108 & 125 & VYPDDKNNLKEPTITGYS & 18 \\
\hline 8 & 151 & 157 & GVNQGGD & 7 \\
\hline$\theta$ & 159 & 170 & NLVNDNPSAVIN & 12 \\
\hline 10 & 174 & 175 & KR & 2 \\
\hline 11 & 188 & 190 & TLADA & 5 \\
\hline 12 & 182 & 193 & GV & 2 \\
\hline 13 & 204 & 213 & LSRPPMPMPI & 10 \\
\hline 14 & 224 & 240 & AAPDNSVPIAAGENSYN & 17 \\
\hline
\end{tabular}

AlgPred: Prediction of Allergenic Proteins and Mapping of IgE Epitopes

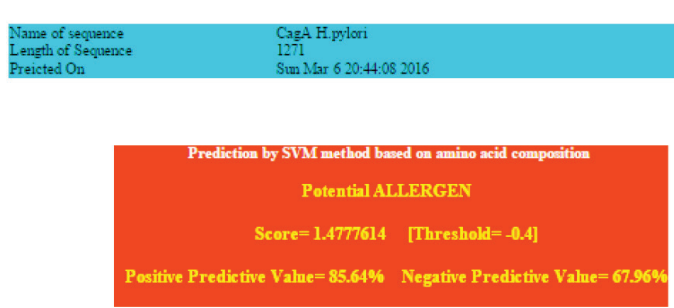

Figure 16. Allergenicity of CagA. against Brucella abortus 544 Strain Infection in BALB/c Mice by Subcutaneouse Administration of Multicomponent Vaccine of rCagA Conjugated with LPS + CpG. J Pure Appl Microbiol. 2013;7(3):1809-1819.

3. Pasquevich KA, Garcia Samartino C, Coria LM, et al. The protein moiety of Brucella abortus outer membrane protein 16 is a new bacterial pathogen-associated molecular pattern that activates dendritic cells in vivo, induces a Th1 immune response, and is a promising self-adjuvanting vaccine against systemic and oral acquired brucellosis. J Immunol. 2010;184(9):5200-5212. doi:10.4049/jimmunol.0902209.

4. Zheng WY, Wang Y, Zhang ZC, Yan F. Immunological characteristics of outer membrane protein omp31 of goat Brucella and its monoclonal antibody. Genet Mol Res. 2015;14(4):11965-11974. doi:10.4238/2015.October.5.10.

5. Ghasemi A, Salari MH, Zarnani AH, et al. Immune reactivity of Brucella melitensis-vaccinated rabbit serum with recombinant Omp31 and DnaK proteins. Iran J Microbiol. 2013;5(1):19-23.

6. Basiri H, Akbari N, Azizpour M, Hosseini SD, Behrozikhah AM, Eskandari S. Amplification, cloning and expression of Brucella melitensis bp26 gene (OMP28) isolated from Markazi province (Iran) and purification of Bp26 Protein. Arch Razi Inst. 2013;68(2):111-116. doi:10.7508/ari.2013.02.004.

7. Yao $\mathrm{L}, \mathrm{Wu} C X$, Zheng $\mathrm{K}$, et al. Immunogenic response to a recombinant pseudorabies virus carrying bp26 gene of Brucella melitensis in mice. Res Vet Sci. 2015;100:61-67. doi:10.1016/j. rvsc.2015.03.030.

8. Cassataro J, Pasquevich K, Bruno L, Wallach JC, Fossati CA, Baldi PC. Antibody reactivity to Omp31 from Brucella melitensis in human and animal infections by smooth and rough Brucellae. Clin Diagn Lab Immunol. 2004;11(1):111-114. doi:10.1128/ cdli.11.1.111-114.2004.

9. Vizcaino N, Cloeckaert A, Zygmunt MS, Dubray G. Cloning, nucleotide sequence, and expression of the Brucella melitensis omp31 gene coding for an immunogenic major outer membrane protein. Infect Immun. 1996;64(9):3744-3751.

10. Ogorodnik E, Raffaniello RD. Analysis of the 3'-variable region of the cagA gene from Helicobacter pylori strains infecting patients at New York City hospitals. Microb Pathog. 2013;56:29-34. doi:10.1016/j.micpath.2012.10.003.

11. Dorneles EM, Sriranganathan N, Lage AP. Recent advances in Brucella abortus vaccines. Vet Res. 2015;46:76. doi:10.1186/ s13567-015-0199-7.

12. Yang X, Skyberg JA, Cao L, Clapp B, Thornburg T, Pascual DW. Progress in Brucella vaccine development. Front Biol (Beijing). 2013;8(1):60-77. doi:10.1007/s11515-012-1196-0.

13. Abkar M, Lotfi AS, Amani J, et al. Survey of Omp19 immunogenicity against Brucella abortus and Brucella melitensis: influence of nanoparticulation versus traditional immunization. Vet Res Commun. 2015;39(4):217-228. doi:10.1007/s11259-015-9645-2.

14. Tabynov K, Sansyzbay A, Kydyrbayev Z, et al. Influenza viral vectors expressing the Brucella OMP16 or L7/L12 proteins as vaccines against B. abortus infection. Virol J. 2014;11:69. doi:10.1186/1743-422x-11-69.

15. Cassataro J, Pasquevich KA, Estein SM, et al. A recombinant subunit vaccine based on the insertion of 27 amino acids from Omp31 to the $\mathrm{N}$-terminus of BLS induced a similar degree of protection against B. ovis than Rev.1 vaccination. Vaccine. 2007;25(22):4437-4446. doi:10.1016/j.vaccine.2007.03.028.

16. Wang W, Wu J, Qiao J, et al. Evaluation of humoral and cellular immune responses to BP26 and OMP31 epitopes in the attenuated Brucella melitensis vaccinated sheep. Vaccine. 2014;32(7):825833. doi:10.1016/j.vaccine.2013.12.028.

17. Amani J, Mousavi Gargari SL, Rafati S, Salmanian AH. In silico analysis of chimeric espA, eae and tir fragments of Escherichia 
coli O157:H7 for oral immunogenic applications. Theor Biol Med Model. 2009;6:28. doi:10.1186/1742-4682-6-28.

18. Amet N, Lee HF, Shen WC. Insertion of the designed helical linker led to increased expression of tf-based fusion proteins. Pharm Res. 2009;26(3):523-528. doi:10.1007/s11095-008-9767-0.

19. Bai $Y$, Shen WC. Improving the oral efficacy of recombinant granulocyte colony-stimulating factor and transferrin fusion protein by spacer optimization. Pharm Res. 2006;23(9):21162121. doi:10.1007/s11095-006-9059-5.

20. Baloria U, Akhoon BA, Gupta SK, Sharma S, Verma V. In silico proteomic characterization of human epidermal growth factor receptor 2 (HER-2) for the mapping of high affinity antigenic determinants against breast cancer. Amino Acids. 2012;42(4):13491360. doi:10.1007/s00726-010-0830-x.

21. Cai H, LiY, Zhang H, Feng F. [Effects of gene design on recombinant protein expression: a review]. Sheng Wu Gong Cheng Xue Bao. 2013;29(9):1201-1213.

22. Klompus S, Solomon G, Gertler A. A simple novel method for the preparation of noncovalent homodimeric, biologically active human interleukin 10 in Escherichia coli-enhancing protein expression by degenerate PCR of $5^{\prime}$ DNA in the open reading frame. Protein Expr Purif. 2008;62(2):199-205. doi:10.1016/j. pep.2008.07.013. 